03

\title{
Влияние числа Рейнольдса на распределение кинетической энергии турбулентных пульсаций по сечению плоского канала
}

\author{
(C) Ю.Г. Чесноков \\ Санкт-Петербургский государственный технологический институт (технический университет), \\ 198013 Санкт-Петербург, Россия \\ e-mail:ygchesnokov@yandex.ru
}

Поступило в Редакцию 8 мая 2018 г.

В окончательной редакции 11 декабря 2018 г.

Принято к публикации 19 декабря 2018 г.

На основе анализа данных, полученных различными авторами методом прямого численного моделирования течения жидкости в плоском канале, изучено влияние числа Рейнольдса на распределение кинетической энергии пульсационного движения по сечению плоского канала.

DOI: 10.21883/JTF.2019.06.47629.195-18

\section{Введение}

Одной из важнейших характеристик турбулентного течения является кинетическая энергия турбулентных пульсаций. Эта величина определяет интенсивность турбулентного перемешивания и такие характеристики, как турбулентная (вихревая) вязкость, коэффициент турбулентной диффузии и т. П. Поэтому закономерности распределения кинетической энергии турбулентных пульсаций в потоке, а также влияние на это распределение числа Рейнольдса, представляет значительный интерес. В инженерных приложениях при расчете турбулентных течений для нахождения кинетической энергии турбулентных пульсации используют модельные уравнения, которые требуют проверки.

Экспериментальное измерение осредненной по времени кинетической энергии турбулентных пульсаций представляет собой сложную задачу. Требуется одновременно замерять все три компоненты пульсационной составляющей скорости, причем для измерений необходимо использовать датчики малого размера. По этой причине при помощи миниатюрных термоанемометров часто измеряют только одну, продольную составляющую скорости. Именно эта составляющая дает главный вклад в кинетическую энергию турбулентных пульсаций. Так, в работе [1] изучалось влияние числа Рейнольдса на среднее по сечению трубы значение кинетической энергии продольных пульсаций скорости жидкости. Продолжают развиваться и оптические методы измерений [2].

Следует отметить, что до настоящего времени отсутствует единая точка зрения на закономерности изменения кинетической энергии пульсационного движения при увеличении числа Рейнольдса. В том случае, когда рассматриваются турбулентные течения, граничащие с твердой поверхностью, такие как течения в пограничном слое, течения в трубе или канале, вводят в рассмотрение так называемые динамическую скорость и динамическую длину. Обозначим через $\tau$ напряжение трения на стенке, через $\rho$ и $v$ плотность и кинематическую вязкость жидкости соответственно. Динамическая скорость $u_{\tau}$ и динамическая длина $l_{\tau}$ определяются при помощи формул

$$
u_{\tau}=\sqrt{\tau / \rho}, l_{\tau}=v / u_{\tau} .
$$

В работе [3] утверждается, что максимальное значение квадрата продольной составляющей пульсационной скорости, отнесенное к квадрату динамической скорости, при увеличении числа Рейнольдса стремится к конечному пределу. Согласно же работе [4], эта величина возрастает пропорционально логарифму числа Рейнольдса, определенного по динамической скорости. Как известно, основной вклад в кинетическую энергию пульсационного движения дают именно пульсации продольной составляющей скорости. Поэтому и кинетическая энергия турбулентных пульсаций при увеличении числа Рейнольдса должна вести себя примерно также как и квадрат продольной составляющей пульсационной скорости.

Детальную информацию о закономерностях распределения кинетической энергии турбулентных пульсаций в зависимости от расстояния до стенки можно получить при помощи результатов, полученных методом прямого численного моделирования. В настоящей работе используются результаты прямого численного моделирования течения в плоском канале, полученные различными авторами [5-15]. Ранее в работах $[16,17]$ результаты, полученные при помощи метода прямого численного моделирования рядом авторов, использовались для изучения влияния числа Рейнольдса на другие статистические характеристики течения, такие, как осредненная скорость, напряжения Рейнольдса и др. В настоящей работе используются данные, полученные для более широкого диапазона значений числа Рейнольдса.

Наибольшее значение кинетической энергии турбулентных пульсаций достигается на небольших расстояниях от стенки, в так называемой буферной зоне. Но даже в этой области в переменных стенки, когда в качестве масштаба длины выбирается динамическая длина, а 
в качестве масштаба скорости динамическая скорость, зависимость кинетической энергии от расстояния до стенки не является универсальной. Цель настоящей работы заключается в изучении влияния числа Рейнольдса на характер этой зависимости. Рассматривается также вопрос о том, какой вклад дают турбулентные пульсации различных направлений в общую кинетическую энергию на различных расстояниях до стенки.

\section{Зависимость кинетической энергии турбулентных пульсаций от расстояния до стенки в переменных стенки}

Будем рассматривать турбулентное течение в плоском канале с полушириной канала $h$. Введем в рассмотрение декартову систему координат $(x, y, z)$, ось $x$ которой направлена по оси канала, ось $y$ по нормали к стенке, а ось $z$ по нормали к плоскости течения. Проекции пульсационной составляющей скорости в продольном, нормальном к стенке и поперечном направлениях будем обозначать через $u, v$ и $w$ соответственно. Введем в рассмотрение безразмерные переменные. Если при переходе к безразмерным переменным в качестве масштаба длины взять динамическую длину, а в качестве масштаба скорости динамическую скорость, то такие переменные называют внутренними или переменными стенками и обозначают при помощи надстрочного индекса „плюс“. Масштабом энергии тогда будет являться величина $\rho u_{\tau}^{2}$. Безразмерную кинетическую энергию турбулентных пульсаций в переменных стенки обозначим через $k^{+}$. Эта величина определяется: $k^{+}=\left(\overline{u^{+2}+v^{+2}+w^{+2}}\right) / 2$. Черта над выражением обозначает применение операции осреднения. Введем в рассмотрение число Рейнольдса, рассчитанное по динамической скорости и полуширине канала:

$$
\operatorname{Re}_{\tau}=\frac{u_{\tau} h}{v}=h^{+}
$$

Эта величина называется также числом Кармана. Из соображений размерности вытекает, что безразмерная кинетическая энергия турбулентных пульсаций $k^{+}$может зависеть только от двух безразмерных величин: безразмерного расстояния до стенки $y^{+}$и числа Кармана $h^{+}$. Обычно считают, что в окрестности стенки при малых значениях $y^{+}$зависимость от $h^{+}$не должна проявляться. Как показывает рис. 1, уже в так называемой буферной зоне, где кинетическая энергия турбулентных пульсаций имеет максимальное значение, графики зависимости $k^{+}$от $y^{+}$при разных значениях $h^{+}$идут поразному. В частности, координата точки, где кинетическая энергия $k^{+}$принимает максимальное значение, зависит от $h^{+}$. Само максимальное значение с ростом $h^{+}$ увеличивается. Обозначим эту координату через $p^{+}$, а максимальное значение через $m^{+}$. Зависимость $p^{+}$от $h^{+}$, построенная по данным, полученным в работах [6-15],

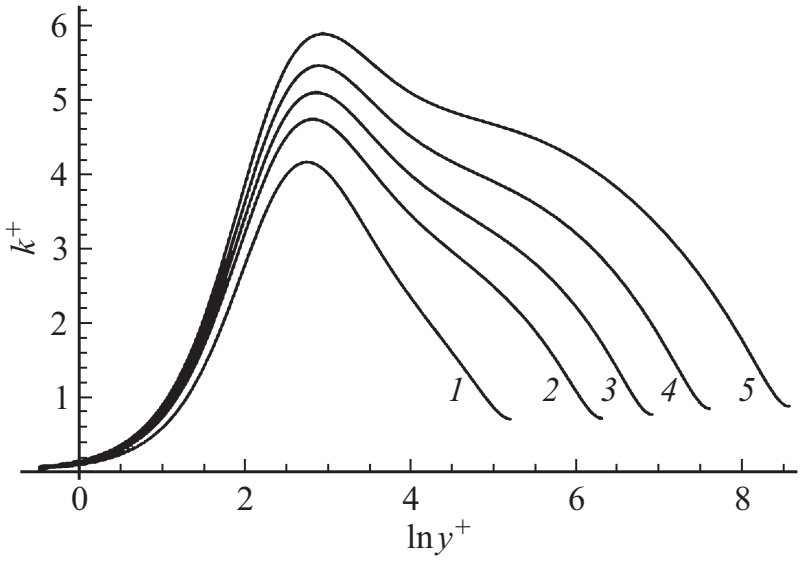

Рис. 1. Зависимости $k^{+}$от $y^{+}$при различных значениях $h^{+}$, построенные по результатам работы [15]. Значения $h^{+}$равны 182 , 543, 1001, 1995 и 5186 (кривые $1-5$ соответственно).

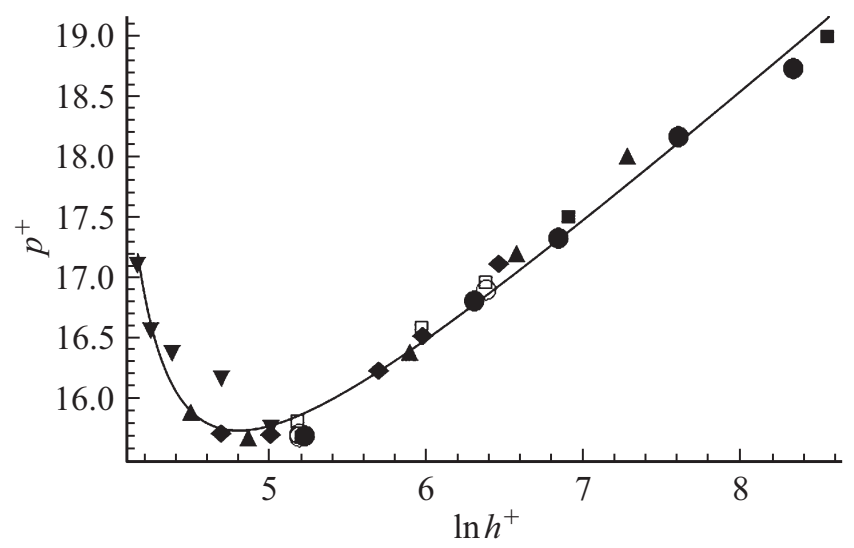

Рис. 2. Зависимость $p^{+}$от $\ln h^{+}$. Кривая построена по формуле (1). Символы обозначают результаты прямого численного моделирования, а именно черные кружки построены по результатам работ $[9,10,13]$, черные квадраты - $[15]$, черные ромбы - [6], черные треугольники вершиной вверх [8], черные треугольники вершиной вниз - [7], светлые диски - $[11,12]$, светлые квадраты - [5].

является немонотонной (рис. 2). Изменяется $p^{+}$при увеличении $h^{+}$очень слабо. Обобщающая формула

$$
p^{+}=9.155+\frac{1.065}{\ln h^{+}-3.835}+1.141 \ln h^{+}
$$

неплохо описывает эту зависимость. Имеются определенные расхождения между результатами различных авторов. Это может быть связано с использованием различных методов решения и систематическими погрешностями численного решения, обусловленными принятым в каждой конкретной работе уровнем дискретизации.

Естественно предполагать, что в непосредственной окрестности стенки при малых значениях $y^{+}$закономерности движения слабо различаются для турбулентных течений различных типов. Для турбулентных течений 


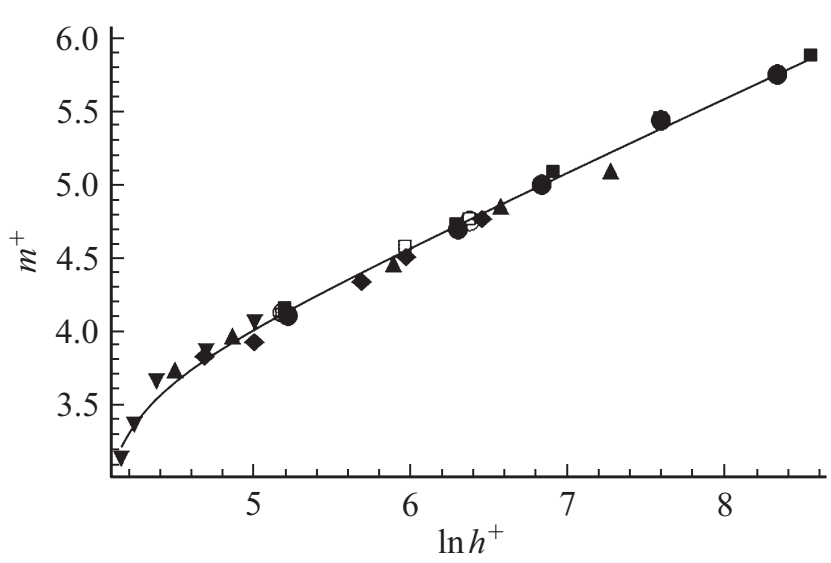

Рис. 3. Зависимость $m^{+}$от $\ln h^{+}$. Обозначения те же, что и на рис. 2. Кривая построена по формуле (2).

в трубе кругового поперечного сечения по данным работы [18] расстояния до стенки, где достигается максимум кинетической энергии, неплохо описывается формулой (1). Данные, полученные для турбулентного пограничного слоя без градиента давления $[19,20]$, показывают, что в этом случае имеются систематические отклонения от зависимости (1). При сравнительно больших значениях $\operatorname{Re}_{\tau}$ значения $p^{+}$получаются больше, чем для плоского канала, но отклонения не превышают 0.5 . За определяющий линейный размер при расчете $\operatorname{Re}_{\tau}$ принимают толщину пограничного слоя. Иначе дело обстоит с турбулентным течением Куэтта в плоском канале. Результаты, полученные в работах [21,22], показывают, что в этом случае и значения $p^{+}$, и значения $m^{+}$ оказываются существенно выше.

Для того чтобы использовать формулу (1), необходимо располагать значением динамической скорости жидкости. Обычно известной величиной является средняя по сечению канала скорость потока. Формулы, которые связывают между собой число Рейнольдса, вычисленное по средней по сечению скорости, и число Рейнольдса, вычисленное по динамической скорости, можно найти в работе [23].

Максимальное значение кинетической энергии турбулентных пульсаций $m^{+}$, как показывает рис. 3, при увеличении числа Кармана $h^{+}$увеличивается. При сравнительно больших значениях $h^{+}$зависимость $m^{+}$от $\ln h^{+}$ близка к линейной. Для расчета можно предложить следующую приближенную формулу:

$$
m^{+}=1.493-\frac{0.04307}{\ln h^{+}-4.0732}+0.516 \ln h^{+} .
$$

При малых значениях $y^{+}$величину $k^{+}$можно разложить в ряд по степеням $y^{+}$. На стенке канала при $y^{+}=0$ в силу условия прилипания пульсационная составляющая скорости обращается в нуль. Поэтому разложение в ряд должно иметь такой вид

$$
k^{+}=a_{1} y^{+2}+a_{2} y^{+3}+\ldots
$$

Данные прямого численного моделирования показывают, что коэффициенты этого разложения зависят от $h^{+}$. Коэффициент $a_{1}$ с ростом числа Кармана монотонно увеличивается. Для описания этой зависимости можно предложить следующую обобщающую зависимость:

$$
a_{1}=0.209-\frac{0.453}{\ln h^{+}-1.45} .
$$

Величину $a_{1}$ можно найти при помощи формул для среднеквадратичных значений проекций пульсационной составляющей скорости из работы [16]. Оказывается, что эти формулы дают неплохие результаты даже при больших значениях $h^{+}$. Наибольшую погрешность эти формулы дают при $h^{+}<100$.

\section{Зависимость кинетической энергии турбулентных пульсаций от числа Рейнольдса во внешней области}

Введем обозначение $Y=y / h$. Такая переменная называется внешней. Как показывают результаты прямого численного моделирования, при фиксированном значении $Y$ зависимость $k^{+}$от $h^{+}$не является монотонной. На рис. 4 показана зависимость кинетической энергии турбулентных пульсаций на оси канала $k_{l}^{+}$от $h^{+}$. При малых значениях числа Рейнольдса эта величина убывает, при $h^{+} \approx 300$ достигается минимальное значение, а затем кинетическая энергия начинает увеличиваться. В целом изменения $k_{l}^{+}$небольшие. Рассчитать эту величину можно при помощи следующей формулы:

$$
k_{l}^{+}=\frac{1.593}{\ln h^{+}-2.76}-0.8991+0.1837 \ln h^{+} .
$$

Такой же характер имеет и зависимость средней по сечению канала кинетической энергии турбулентных пульсаций от числа Рейнольдса. В работе [1] исследовалась зависимость от числа Рейнольдса величины $\overline{u^{2}} / 2$, усредненной по поперечному сечению трубы кругового поперечного сечения. Обозначим через $\operatorname{Re}$ число

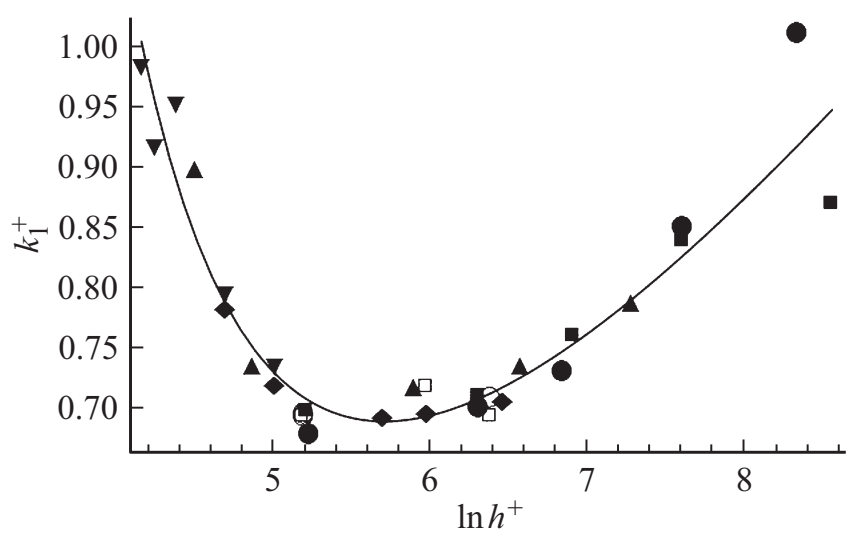

Рис. 4. Зависимость $k_{l}^{+}$от $\ln h^{+}$. Обозначения те же, что и на рис. 2. Кривая построена по формуле (3). 
Рейнольдса, определенное по средней по поперечному сечению трубы скорости и диаметру трубы. Согласно $[1]$, при $2.4 \cdot 10^{4}<\operatorname{Re}<10^{5}$ кинетическая энергия пропорциональна квадрату средней по сечению скорости, т.е. отношение этой величины к квадрату средней по сечению скорости не зависит от числа Рейнольдса. При $\operatorname{Re}>10^{5}$ характер зависимости изменяется. По данным [1] в этой области кинетическая энергия пропорциональна квадрату динамической скорости. Для течения в плоском канале данные прямого численного моделирования не подтверждают эти выводы. Обозначим через $U_{m}$ среднюю по поперечному сечению канала скорость жидкости, а через $K^{+}=\int_{0}^{1} k^{+} d Y-$ среднюю по сечению канала кинетическую энергию пульсационного движения. Величина $K^{+} / U_{m}^{+2}$ при увеличении числа Рейнольдса монотонно убывает. Эта зависимость неплохо описывается при помощи следующего обобщающего соотношения:

$$
\frac{K^{+}}{U_{m}^{+2}}=0.001206+\frac{0.01758}{\ln h^{+}-2.284} .
$$

С другой стороны, при сравнительно больших значениях числа Рейнольдса величина $K^{+}$не сохраняет постоянное значение. При помощи результатов работ [5-15] можно получить обобщающую зависимость

$$
K^{+}=\frac{0.2711}{\ln h^{+}-3.967}+0.5193+0.1999 \ln h^{+} .
$$

\section{Вклад продольной, нормальной к стенке и поперечной составляющих пульсационной скорости в кинетическую энергию пульсационного движения}

Как известно, для течений в трубах и каналах характерна анизотропия распределения пульсационной со-

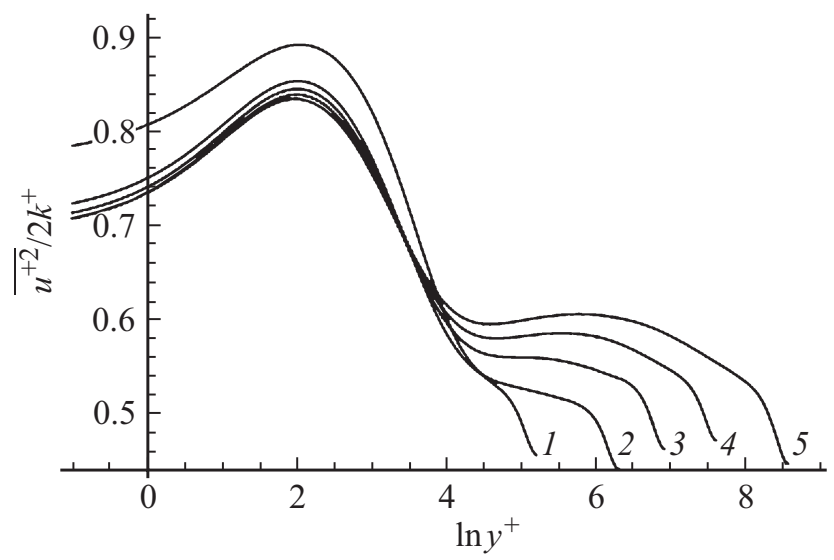

Рис. 5. Зависимости $\overline{u^{+2}} / 2 k^{+}$от $y^{+}$при различных значениях $h^{+}$, построенные по результатам работы [15]. Значения $h^{+}$ равны 182, 543, 1001, 1995 и 5186 (кривые 1-5 соответственно).

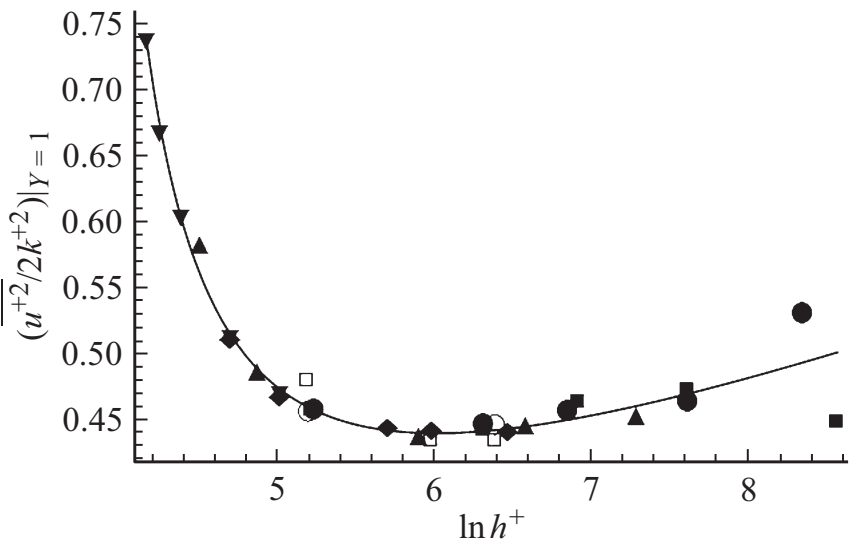

Рис. 6. Зависимость $\left(\overline{u^{+2}} / 2 k^{+2}\right)_{Y=1}$ от $\ln h^{+}$. Обозначения те же, что и на рис. 2. Кривая построена по формуле (4).

ставляющей скорости. Продольные пульсации скорости дают основной вклад в кинетическую энергию пульсационного движения. О роли различных составляющих пульсационной скорости можно судить по численным значениям величин $\overline{u^{+2}} /\left(2 k^{+}\right), \overline{v^{* 2}} /\left(2 k^{+}\right)$и $\overline{w^{+2}} /\left(2 k^{+}\right)$. Рис. 5 показывает, что при всех значениях числа Рейнольдса во всей области движения продольная составляющая пульсационной скорости дает наибольший вклад в кинетическую энергию. Наибольшие значения величина $\overline{u^{+2}} /\left(2 k^{+}\right)$имеет в окрестности стенки канала. В этой пристенной области влияние числа Рейнольдса является заметно выраженным. При увеличении числа Рейнольдса наблюдается некоторая тенденция к уменьшению анизотропии. Непосредственно на стенке величина $\overline{u^{+2}} /\left(2 k^{+}\right)$ монотонно уменьшается по мере увеличения числа Рейнольдса. Эту зависимость можно описать при помощи соотношения

$$
\left.\overline{\frac{u^{+2}}{2 k^{+}}}\right|_{y^{\prime}=0}=0.6554+\frac{48.53}{\left(\ln h^{+}\right)^{3.68}}
$$

Максимальное значение величины $\overline{u^{+2}} /\left(2 k^{+}\right)$достигается вблизи стенки при $y^{+}=p_{u}^{+}$. Обозначим это максимальное значение через $m_{u}^{+}$. Точка, где достигается максимум, в переменных стенки с ростом число Рейнольдса медленно смещается в сторону стенки, но остается в пределах так называемой буферной зоны. Справедлива следующая обобщающая формула:

$$
p_{u}^{+}=7.092+\frac{1.047}{\ln h^{+}-3.622} .
$$

Зависимость $m_{u}^{+}$от числа Рейнольдса описывается при помощи соотношения

$$
m_{u}^{+}=0.8059+\frac{8.87}{\left(\ln h^{+}\right)^{2.81}} .
$$

Во внешней области при увеличении числа Рейнольдса изменяется характер зависимости $\overline{u^{+2}} / k^{+}$величины от расстояния до стенки. Как показывает рис. 5, 
при сравнительно больших значения числа Рейнольдса появляются два локальных экстремума (минимум и максимум). На оси канала при сравнительно малых значениях $h^{+}$величина $\overline{u^{+2}} /\left(2 k^{+}\right)$при $Y=1$ с ростом $h^{+}$ сначала довольно быстро уменьшается (рис. 6). Затем проявляется тенденция к медленному увеличению этой величины. Формула для описания этой зависимости такова

$$
\left.\frac{\overline{u^{+2}}}{\left(2 k^{+}\right)}\right|_{Y=1}=0.2917+\frac{134400}{\left(\ln h^{+}\right)^{9.03}}+0.02287 \ln h^{+} .
$$

Рис. 7 показывает, что поперечная составляющая скорости дает наименьший вклад в энергию пульсационного движения в точке, расположенной в буферной зоне. Обозначим координату этой точки через $p_{w}^{+}$, а минимальное значение величины $\overline{w^{+2}} /\left(2 k^{+}\right)$через $m_{w}^{+}$. При одинаковых значениях числа Рейнольдса точка, где достигается минимальное значение величины $\overline{w^{+2}} /\left(2 k^{+}\right)$, располагается немного дальше от стенки, чем точка с координатой $p_{u}^{+}$. Формула для расчета $p_{w}^{+}$имеет вид

$$
p_{w}^{+}=8.036+\frac{1.155}{\ln h^{+}-3.62} .
$$

В переменных стенки с ростом числа Рейнольдса точка, где достигается минимум, медленно смещается в сторону стенки. Зависимость минимального значения от числа Рейнольдса описывается при помощи следующего соотношения:

$$
m_{w}^{+}=0.179-\frac{7.993}{\left(\ln h^{+}\right)^{2.798}}
$$

Поскольку на стенке канала величина $\overline{v^{+2}} /\left(2 k^{+}\right)$обращается в нуль, а величина $\overline{u^{2 k^{+}}} /\left(2 k^{+}\right)$с ростом числа Рейнольдса уменьшается, величина $\overline{w^{+2}} /\left(2 k^{+}\right)$на стенке с ростом числа Рейнольдса увеличивается. Во внешней области при различных значениях числа Рейнольдса

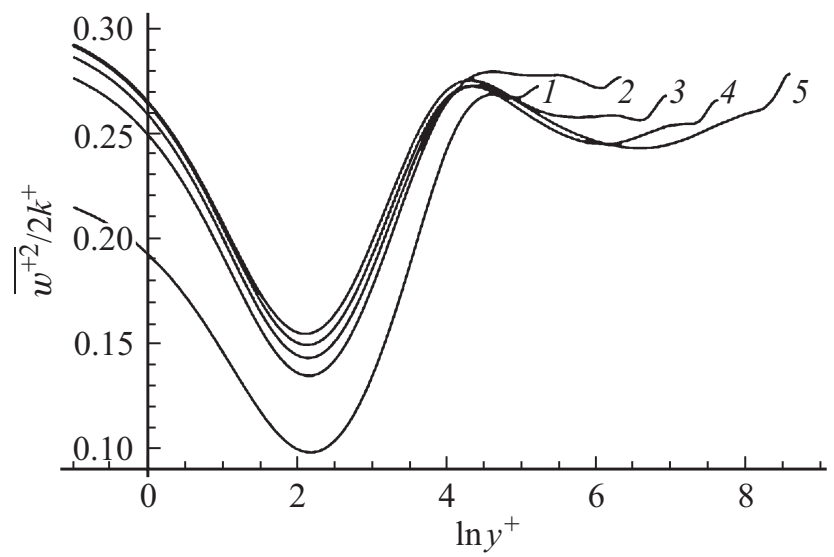

Рис. 7. Зависимости $\overline{w^{+2}} / 2 k^{+}$от $y^{+}$при различных значениях $h^{+}$, построенные по результатам работы [15]. Значения $h^{+}$ равны 182, 543, 1001, 1995 и 5186 (кривые 1-5 соответственно).

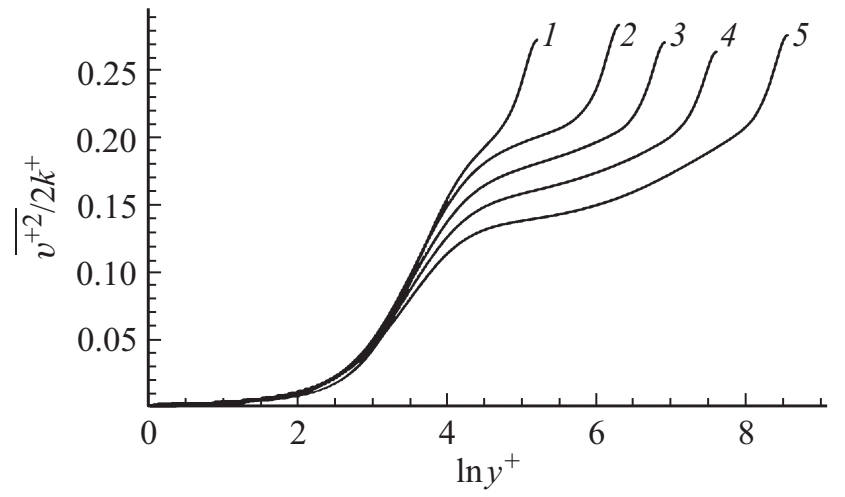

Рис. 8. Зависимости $\overline{v^{+2}} / 2 k^{+}$от $y^{+}$при различных значениях $h^{+}$, построенные по результатам работы [15]. Значения $h^{+}$ равны 182, 543, 1001, 1995 и 5186 (кривые 1-5 соответственно).

зависимости $\overline{w^{+2}} /\left(2 k^{+}\right)$от $y^{+}$имеют локальные экстремумы (максимум и минимум). На оси канала величина $\overline{w^{+2}} /\left(2 k^{+}\right)$от $y^{+}$при малых числах Рейнольдса быстро увеличивается при увеличении числа Рейнольдса, а затем медленно убывает. В рассматриваемом диапазоне значений числа Рейнольдса эта зависимость такова:

$$
\left.\frac{\overline{w^{+2}}}{2 k^{+}}\right|_{Y=1}=0.3534-\frac{0.03883}{\ln h^{+}-3.907}-0.009913 \ln h^{+} .
$$

На стенке канала в нуль обращается не только сама нормальная к стенке проекция пульсационной скорости $v^{+}$, но и (в силу уравнения неразрывности) ее производная по $y^{+}$. Поэтому величина $\overline{v^{+2}} /\left(2 k^{+}\right)$при малых $y^{+}$является малой. Как показывает рис. 8 , она монотонно увеличивается при увеличении расстояния до стенки. При малых $y^{+}$зависимость величины $\overline{v^{+2}} /\left(2 k^{+}\right)$ от числа Рейнольдса весьма слабая.

\section{Заключение}

Полученные в работе результаты позволяют судить о том, какое влияние число Рейнольдса оказывает на изменение кинетической энергии турбулентных пульсаций по сечению плоского канала. Точка, где кинетическая энергия пульсационного движения является максимальной, лежит в буферной области и в переменных стенки медленно смещается от стенки при увеличении числа Рейнольса, рассчитанного по динамической скорости жидкости. Исключение составляют малые значения этого числа. С ростом числа Рейнольдса максимальное значение кинетической энергии увеличивается примерно пропорционально логарифму числа Рейнольдса. Во внешней области при фиксированном значении $Y$ зависимость кинетической энергии от числа Рейнольдса является немонотонной. Во всей области движения продольные пульсации скорости дают наибольший вклад в кинетическую энергию пульсационного движения, но 
наибольший вклад они дают в вязком подслое и буферной зоне. В буферной зоне располагается и точка, где вклад поперечных пульсаций скорости в кинетическую энергию является наименьшим. При увеличении числа Рейнольдса анизотропия распределения пульсационной скорости в этой области уменьшается.

\section{Список литературы}

[1] Yakhot V., Bailey S.C.C., Smits A.J. // J. Fluid. Mech. 2010. Vol. 652. P. 65-73.

[2] Schneiders J.F.G., Scarano F., Elsinga G.E. // Exp. Fluid. 2017. Vol. 58. P. 27.

[3] Monkewitz P.A., Nagib H.M. // J. Fluid. Mech. 2015. Vol. 783. P. 474-503.

[4] Marusic I., Baars W.J., Hutchins N. // Phys. Rev. Fluid. 2017. Vol. 2. P. 100502.

[5] Moser R.D., Kim J., Mansour N.N. // Phys. Fluid. 1999. Vol. 11. N 4. P. $943-945$.

[6] Iwamoto K., Suzuki S., Kasagi N. // Int. J. Heat Fluid. Flow. 2002. Vol. 23. P. 678-689.

[7] Tsukahara T., Seki Y., Kawamura H., Tochio D. In: Proc. of the Forth. Int. Symp. on Turbulence and Shear Flow Phenomena. Williamsburg, USA. 2005. P. 935-940.

[8] Hu Z.W., Morfey C.L., Sandham N.D. // AIAA J. 2006. Vol. 44. N 2. P. 1541-1549.

[9] Hoyas S., Jiménez J. // Phys. Fluid. 2006. Vol. 18. P. 011702.

[10] Hoyas S., Jiménez J. // Phys. Fluid. 2008. Vol. 20. P. 101511.

[11] Wreman A.W., Kuerten J.G.M. // Phys. Fluid. 2014. Vol. 26. P. 015102.

[12] Wreman A.W., Kuerten J.G.M. // Phys. Fluid. 2014. Vol. 26. P. 085103.

[13] Lozano-Duran A., Jiménez J. // Phys. Fluid. 2014. Vol. 26. P. 011702.

[14] Bernardini M., Pirozzoli S., Orlandi P. // J. Fluid. Mech. 2014. Vol. 742. P. 171-191.

[15] Lee M., Mozer R.D. // J. Fluid. Mech. 2015. Vol. 774. P. 395-415.

[16] Чесноков Ю.Г. // ЖТФ. 2010. Т. 80. Вып. 12. С. 33-39. [Chesnokov Y.G. // Tech. Phys. 2010. Vol. 55. N 12. P. 1741-1747.]

[17] Чесноков Ю.Г. // ЖТФ. 2011. Т. 81. С. 30-34. [Chesnokov Y.G. // Tech. Phys. 2011. Vol. 57. N 7. P. 931-935.]

[18] El Khouri G.K., Schlatter P., Noorani A., Fischer P.F., Brethouwer G., Johanson A.V. // Flow Turbul. Combust. 2013. Vol. 91. P. 475-495.

[19] Jimenéz J., Hoyas S., Simens M.P., Mizuno Y. // J. Fluid. Mech. 2010. Vol. 657. P. 335-360.

[20] Sillero J.A., Jimenéz J., Moser R.D. // Phys. Fluid. 2013. Vol. 25. P. 105102.

[21] Avsarkisov V., Hoyas S., Oberlack M., Galache G.P.G. // J. Fluid. Mech. 2014. Vol. 751. R1.

[22] Pirozzoli S., Bernardini M., Orlandi P. // J. Fluid. Mech. 2014. Vol. 758. P. 327-343.

[23] Чесноков Ю.Г. // Изв. СПбГТИ (ТУ). 2016. № 36. C. 104-107. 